\title{
Road state estimation based on acoustic analysis
}

\section{Estimación del estado del asfalto mediante análisis acústico}

\author{
Jesús Alonso • Juan Manuel López • Ignacio Pavón • \\ César Asensio • Guillermo De Arcas \\ (C) Etrasa 2014
}

\begin{abstract}
An electronic system capable of estimating the road surface status in real time, and among dry/wet/ icy/snowy is shown. The system is mainly based on the analysis of the tyre/road noise generated during vehicle driving. The sound emission pattern of the tyre/road interaction changes dramatically depending on whether the pavement is dry, wet, icy or snowy. Thus discriminating the tyre/road noise acoustic footprint, it is possible to estimate the road status. To be able to distinguish this acoustic footprint, a Support Vector Machines (SVM) based classifier has been developed. Tyre/road noise is initially captured using a properly conditioned microphone. The obtained signal is digitized and preprocessed, extracting its spectral components to produce the feature vector that will be fed to classifier. Finally the classifier estimates the condition of the asphalt, which may be broadcast through the vehicle communication interface, to be shown on the console, or to be used by other subsystems in order to improve safety and comfort. The connection to the vehicle engine control units through its communications interface, also allows obtaining information on its dynamic variables, allowing improving the results obtained by the classifier. Before the system is able to operate properly, the classifier needs to be trained. In initial tests, the system has been trained to distinguish the states of dry and wet
\end{abstract}

J. Alonso $(\bowtie) \cdot J$ J. M. López · I. Pavón • C. Asensio •

G. De Arcas

Grupo de Investigación en Instrumentación y Acústica

Aplicada, Universidad Politécnica de Madrid,

Madrid, Spain

e-mail: jesus.alonsof@upm.es asphalt, yielding success rates around $91 \%$ and an average response time in the transition from dry to wet of about $0.2 \mathrm{~s}$. These results demonstrate the feasibility of the system. Currently, a reference implementation of the system is under development. It integrates all the elements needed in a board of reduced size and low cost. Current implementation of the system could improve security by issuing a slippery asphalt warning, when it detects wet asphalt. Data obtained may also be used by the traction control subsystem to improve safety and comfort. Additional work is being made to extend the classifier algorithms to detect icy and snowy asphalt states.

Resumen En este trabajo, se presenta un sistema electrónico para estimar el estado del asfalto en tiempo real entre cuatro posibles situaciones: seco, mojado, hielo y nieve. El sistema se basa principalmente en el análisis del ruido de rodadura generado durante la marcha del vehículo. El patrón de emisión sonora de la interacción neumático pavimento cambia notablemente dependiendo de si el asfalto está seco, mojado, cubierto de hielo o cubierto de nieve. Por tanto, discriminando la huella acústica del ruido de rodadura, es posible determinar el estado del asfalto. Para poder distinguir esta huella acústica, se ha desarrollado un clasificador, basado en máquinas de vectores soporte (Support Vector Machines o SVM). El ruido de rodadura es captado inicialmente por un micrófono debidamente acondicionado. La señal obtenida es digitalizada y preprocesada, extrayéndose de ella sus componentes espectrales para elaborar el vector de características 
que usará el clasificador. Este clasificador finalmente realiza una estimación del estado del asfalto, que podrá ser difundida a través de la interfaz de comunicaciones del vehículo, para ser mostrada en la consola del mismo, o para que sea utilizada por otros subsistemas con el fin de mejorar la seguridad y confort. La conexión con las centralitas del vehículo a través del interfaz de comunicaciones del mismo, también permite obtener información de las variables dinámicas, permitiendo mejorar los resultados obtenidos por el clasificador. Antes de que el sistema pueda funcionar correctamente, el clasificador ha de ser entrenado. En las pruebas iniciales, el sistema ha sido entrenado para distinguir los estados de asfalto seco y mojado, obteniéndose tasas de acierto alrededor del $91 \%$, y un tiempo medio de respuesta en la transición de seco a mojado de unos 0,2 s. Estos resultados demuestran la viabilidad del sistema. Actualmente se está desarrollando una implementación de referencia en forma de prototipo de tamaño reducido que integra todos los elementos necesarios del sistema en una tarjeta de reducidas dimensiones y bajo coste. La implementación actual del sistema podría mejorar la seguridad mediante la emisión de un aviso de asfalto resbaladizo, cuando se detecta una condición de asfalto mojado. Los datos obtenidos podrían ser utilizados también por el subsistema de control de tracción para mejorar la seguridad y confort. Adicionalmente se está trabajando en extender la detección a los estados de asfalto helado y asfalto nevado.

Keywords New in-vehicle technologies · Prevention . Intelligent transportation - Tyre/road noise - Support vector machines

Palabras clave Nuevas tecnologías en vehículo . Prevención · Transporte inteligente · Ruido de rodadura . Máquinas de vectores soporte

\section{Introducción}

Estado climático del asfalto y seguridad

Cuando se analizan las estadísticas de accidentes de vehículos a motor, la climatología juega un papel importante. Las inclemencias del tiempo producen alteraciones en el estado del firme, que modifican su agarre. Cuando el asfalto está mojado, su adherencia disminuye. Si la acumulación de agua alcanza una determinada profundidad, puede producirse incluso una pérdida completa del control del vehículo debida al efecto de aquaplaning. El asfalto nevado o helado es igualmente peligroso, especialmente algunos casos en los que se produce una delgada y transparente capa de hielo sobre la carretera, muy resbaladiza y difícil de ver.

En los casos en los que el conductor no se percata del estado del asfalto mojado, nevado o helado, el aumento en el riesgo asociado a la conducción es obvio. Pero aun en los casos en los que el conductor se percata del peligro, diversos estudios ponen en evidencia su capacidad para ajustar la conducción a estas nuevas condiciones [4, 8]. Esto se debe a diversos factores psicológicos, como el conocido efecto anclaje [1], que se refiere a la dificultad que tenemos los seres humanos para cambiar una hipótesis inicial incluso a pesar de la aparición a posteriori de evidencias en su contra. La apreciación que el conductor tiene de la validez de las distintas señales que le advierten de distintos peligros, también juega un factor importante, habiendo demostrado las señales variables una mayor efectividad que las fijas $[2,5,6]$.

Una mejora en la percepción que el conductor tiene de los peligros debidos a las diversas condiciones climáticas, podría mejorar la adaptación de su estilo de conducción a las mismas, mejorando la seguridad en la conducción. Por lo tanto podría ser beneficiosa la existencia de un sistema que advirtiese al conductor de forma fiable y en tiempo real, acerca del estado climático del asfalto. Este sistema debería ubicarse en el propio vehículo a motor, para informar al conductor acerca del estado del trayecto por el que circula. Además, este sistema podría volcar la información en el bus de comunicaciones del vehículo, permitiendo que otros subsistemas, como por ejemplo el control de tracción, utilicen los datos con el fin de mejorar la seguridad y el confort.

\section{Descripción del sistema}

El sistema propuesto para detectar la condición climática del asfalto se basa en el análisis de la huella acústica producida por la interacción rueda/asfalto durante la conducción del vehículo a motor. Esta huella acústica difiere dependiendo del estado del asfalto, siendo distinto el ruido de rodadura producido cuando el vehículo circula sobre pavimento seco, que el producido cuando circula por pavimento mojado, 
helado o nevado. La Figura 1 muestra el ruido de rodadura obtenido durante una prueba en la que el vehículo pasa de circular por un segmento de asfalto seco, a otro segmento mojado, y luego de nuevo a un segmento de asfalto seco. Se puede apreciar que la transición es claramente visible, con un incremento considerable del ruido, especialmente en frecuencias bajas.

El análisis de la huella acústica generada por la interacción rueda/asfalto, se ha realizado extrayendo las características espectrales de la misma, en forma de bandas de 1/3 de octava, para luego alimentarlas a un clasificador basado en máquinas de vectores soporte (SVM, Support Vector Machine).

\section{Sistema propuesto}

El sistema propuesto para realizar la estimación en tiempo real del estado del asfalto, se compone de los bloques mostrados en la Figura 2. En primer lugar, el sistema dispone de un micrófono, que deberá estar ubicado cerca de la rueda para captar el ruido de rodadura de la misma. Dado que el micrófono es un elemento sensible, deberá también protegerse adecuadamente. Un lugar adecuado para la ubicación del micrófono es generalmente en el parachoques trasero del vehículo, en el lado opuesto al que contiene el tubo de escape. No obstante distintos vehículos pueden presentar otros lugares donde la ubicación del micrófono sea más ventajosa.

La señal eléctrica que el micrófono genera a partir del ruido de rodadura, es recogida por un módulo de adquisición de datos, que se encarga de amplificarla debidamente, para luego transformarla en una señal digital. Esta señal digital que contiene la información del ruido de rodadura capturado, es entregada al módulo de extracción de características, que agrupa las muestras de audio en bloques de una duración determinada. Después para cada bloque de muestras, normaliza la señal y extrae las características frecuenciales de interés, en forma de niveles de bandas de 1/3 de octava. Estas componentes frecuenciales son entregadas al clasificador, que las evaluará con el fin de estimar el estado del asfalto.

El bloque clasificador implementa un clasificador basado en máquinas de vectores soporte (SVM). En la actualidad existen muy diversos tipos de clasificadores que podrían haberse utilizado para realizar esta tarea, como por ejemplo los bayesianos, los basados en árboles de decisión, las redes neuronales, etc. Sin embargo se ha decidido usar un clasificador de tipo SVM porque son fáciles de implementar y entrenar, y su tasa de aciertos cuando se utilizan datos que están fuera del conjunto de entrenamiento suele ser superior a la de los anteriormente mencionados [7, Sec. 3.7.1].

Cuando se diseñan clasificadores, se pretende obtener un algoritmo que separe una serie de elementos en distintos grupos (o clases) $\omega_{i}$, en función de distintas características de estos elementos, que conforman los vectores de características $x_{i}$. Los clasificadores SVM son de tipo supervisado, por lo que requieren una fase de entrenamiento para funcionar correctamente. En la fase de entrenamiento, se obtiene una serie de vectores de características $x_{i}$, así como la clase $y_{i}$ que corresponde a cada vector de características obtenido. Para el caso de dos clases, $y_{i}$ tomará el valor 1 para la clase $\omega_{1}, \mathrm{y}-1$ para la clase $\omega_{2}$. La Figura 3 muestra un ejemplo típico de los datos obtenidos en la fase de entrenamiento, para el caso de dos clases, y para vectores de características de dos dimensiones. En esta figura, se han representado dos casos a) y b). En ambos casos, las clases $\omega_{1}$ y $\omega_{2}$ están separadas perfectamente por una línea (que de ahora en adelante en lugar de línea, denominaremos hiperplano para generalizar a $N$ dimensiones).

En la Figura 3a, el hiperplano que separa ambas clases, deja un margen de separación de clases igual a $2 \cdot z_{2}$, mientras que en la Figura $3 \mathrm{~b}$ el margen de separación de clases es $2 \cdot z_{1}$. Dado que $z_{1}>z_{2}$, el margen de separación de clases en la Figura $3 b$ es mayor, y por tanto, si bien ambos clasificadores tienen un rendimiento del $100 \%$ para clasificar los datos del conjunto de entrenamiento, se espera que el clasificador implementado usando el hiperplano mostrado en la Figura $3 b$ tenga mejor rendimiento cuando tenga que clasificar elementos que se encuentran fuera del conjunto de entrenamiento. En general, el rendimiento a la hora de clasificar datos fuera del conjunto de entrenamiento, será tanto mayor cuanto mayor sea el margen de separación de clases. Si bien algoritmos de clasificación como por ejemplo las redes neuronales, podrían mostrar soluciones como la de la Figura 3a, los clasificadores basados en SVM buscan siempre el máximo margen de separación de clases. Para lograrlo, obtienen el hiperplano óptimo ajustándolo únicamente a los elementos críticos que más cerca están de los de la otra clase: los vectores soporte. Para el caso del ejemplo, estos vectores soporte son los que aparecen rodeados 
Figura 1 Huella acústica durante una transición de asfalto seco/mojado/seco. El eje de ordenadas representa el tiempo en unidades de $125 \mathrm{~ms}$

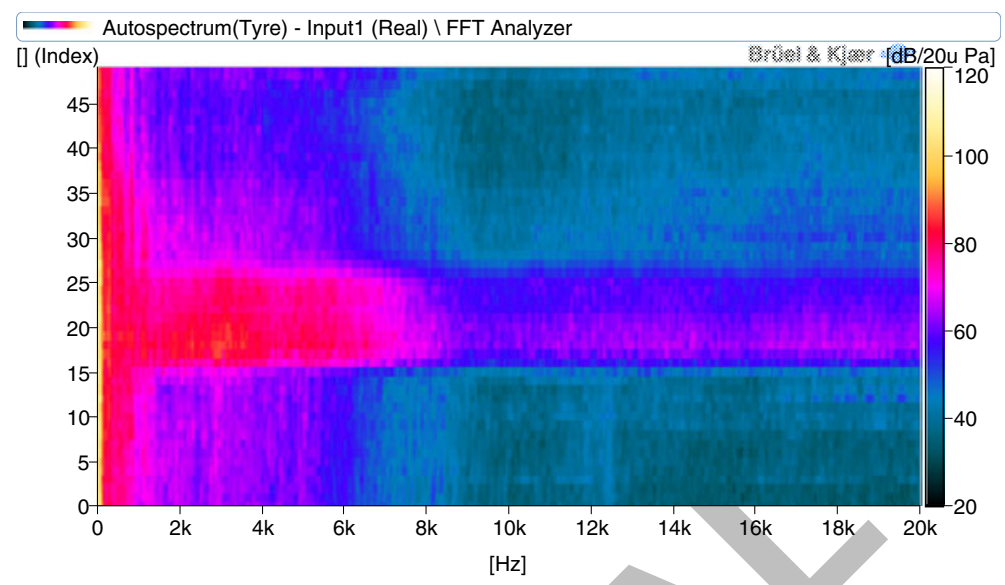

por un círculo en la Figura 3b. Para obtener este hiperplano, es necesario resolver el siguiente problema de optimización:

$$
\begin{gathered}
\min _{w, b, \xi} \frac{1}{2} w^{T} w+C \sum_{i=1}^{l} \xi_{i} \\
y_{i}\left(w^{T} \varphi\left(x_{i}\right)+b\right) \geq 1-\xi_{i}, \quad \xi_{i} \geq 0
\end{gathered}
$$

La función $\varphi\left(x_{i}\right)$ y sirve para mapear los vectores de entrenamiento $x_{i}$ en un espacio dimensional mayor, permitiendo una separación de clases que en principio puedan ser no linealmente separables. A la función $K\left(x_{i}\right.$, $\left.x_{j}\right) \equiv \varphi\left(x_{i}\right)^{T} \varphi\left(x_{j}\right)$, se la conoce como función kernel. Si bien existen diversos tipos de función kernel típicamente utilizadas en SVM, para la implementación de este sistema se ha utilizado la más sencilla, el kernel lineal, cuya expresión es: $K\left(x_{i}, x_{j}\right)=x_{i}{ }^{T} x_{j}$. El parámetro $C$, ha de ser mayor o igual a 0 , y es el término de error permitido al clasificador. En los casos en los que las clases no son separables, este parámetro controla la influencia relativa de los términos que compiten, haciendo no separables a las clases.

Para que los algoritmos SVM tengan un rendimiento óptimo, es necesario realizar una correcta selección de las características que se introducen en el clasificador. Reducir el número de características en un clasificador
SVM generalmente aporta dos beneficios: en primer lugar, reduce la potencia de computación requerida, y en segundo lugar suele mejorar la tasa de aciertos del clasificador. Para reducir el número de características utilizadas, se han probado dos algoritmos: el de eliminación recursiva de características (Recursive Feature Elimination o RFE) [3], y el de minimización de la norma-cero (zero-norm minimization o L0) [9].

Para realizar el entrenamiento del clasificador y para implementar los algoritmos de selección de características, se han utilizado las herramientas Spider [10] de Matlab, que implementan los algoritmos necesarios para realizar todos estos procesos. Las primeras pruebas efectuadas sobre el clasificador arrojaron datos prometedores, aunque aparecieron dos problemas que reducían la tasa de acierto:

1. A velocidades por debajo de los $30 \mathrm{~km} / \mathrm{h}$, el detector tiene una tendencia elevada a generar falsos positivos (es decir, a estimar estado de asfalto mojado aun cuando el firme esté seco). En principio dado que a velocidades bajas, el asfalto mojado no presenta un peligro importante, se ha optado por desactivar el clasificador a estas velocidades.

2. Eventos espurios como los causados por ejemplo por pequeñas piedras impactando en las cercanías del micrófono, pueden hacer que el sistema estime una clasificación errónea, por lo que se ha añadido

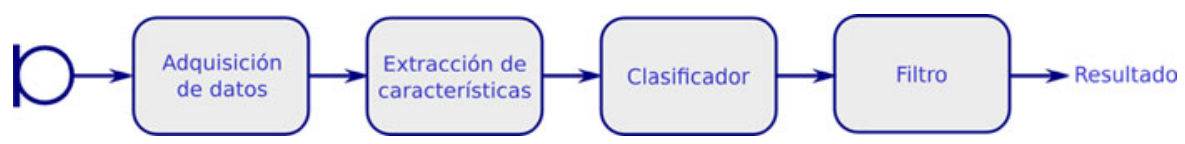

Figura 2 Diagrama de bloques del sistema 

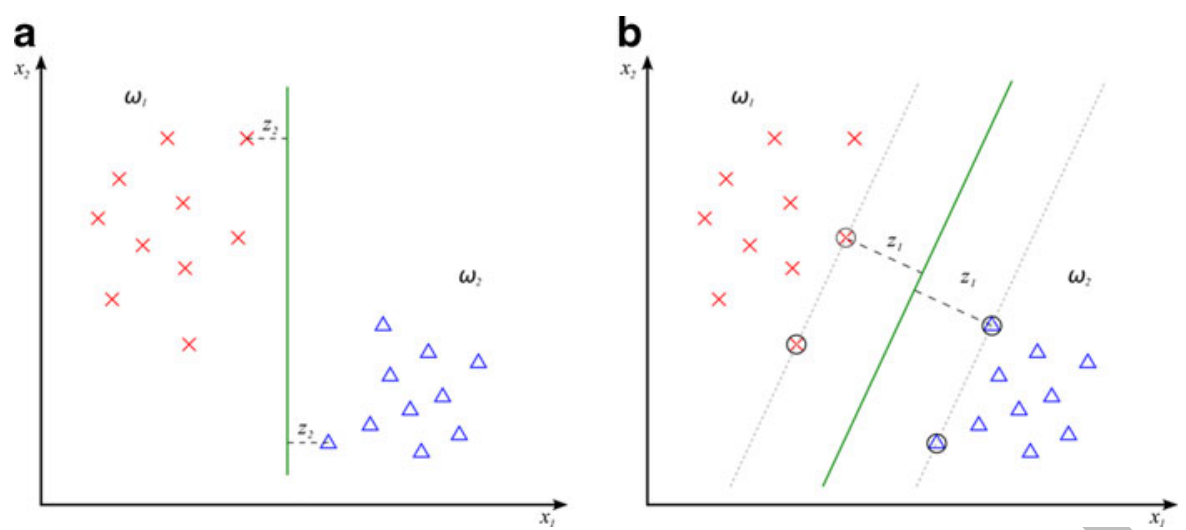

Figura 3 Posibles hiperplanos para separar dos clases. a sin restricción; b con máximo márgen de separación de clases

un bloque de filtrado tras el clasificador, cuyo objetivo es minimizar el efecto de estos eventos espurios de muy corta duración.

\section{Pruebas experimentales}

En una primera fase de pruebas, el clasificador completo ha sido implementado en Matlab, para realizar el entrenamiento y pruebas iniciales del sistema. En un principio se ha restringido el número de clases que el equipo es capaz de detectar a 2: asfalto seco y asfalto mojado. Para entrenar al clasificador, se ha realizado una primera sesión de grabación de ruido de rodadura en un circuito cerrado, haciendo circular al vehículo de pruebas sobre asfalto con secciones de asfalto seco y mojado. $\mathrm{Si}$ bien la captura y grabación de datos se ha realizado con un front-end de Pulse, dado el enfoque práctico que se le quiere dar al sistema, el micrófono utilizado en estas grabaciones ha sido un simple micrófono electret modelo WM-63PR de Panasonic (que puede encontrarse a precios alrededor de $1 €)$. Con los datos obtenidos en esta primera sesión, se ha elaborado el vector de entrenamiento, que se ha utilizado para realizar la selección de características y el entrenamiento del sistema. En varios días posteriores, se ha repetido el experimento, realizando grabaciones sobre asfalto seco y mojado en el mismo circuito, con el fin de construir el vector de pruebas. Este vector de pruebas ha sido aplicado al clasificador construido en Matlab, obteniéndose datos muy prometedores, con tasas de acierto del $100 \%$ para detección de la transición de asfalto seco a asfalto mojado, y del $91 \%$ para la detección de la transición de asfalto mojado a asfalto seco, para velocidades superiores a $30 \mathrm{~km} / \mathrm{h}$. El tiempo de respuesta medio obtenido para la detección de asfalto mojado, ha sido de alrededor de $0,2 \mathrm{~s}$ (si bien el margen de error en la medida de este tiempo de respuesta es difícil de determinar).

A la vista de los buenos resultados obtenidos usando el método de grabación y post-procesado del ruido de rodadura, se decidió implementar un prototipo inicial que permitiese realizar la detección del estado del asfalto con un sistema construido a medida. Para ello se elaboró un hardware consistente en un sistema de adquisición equipado con dos canales de medida, un interfaz para la conexión del sistema al bus de comunicaciones del vehículo usando el estándar OBD-II, y un procesador basado en DSP de punto flotante. Este equipo puede verse en la parte izquierda de la Figura 4. En la parte
Figura 4 Prototipo inicial del sistema de detección de estado del asfalto, y ubicación del micrófono de pruebas

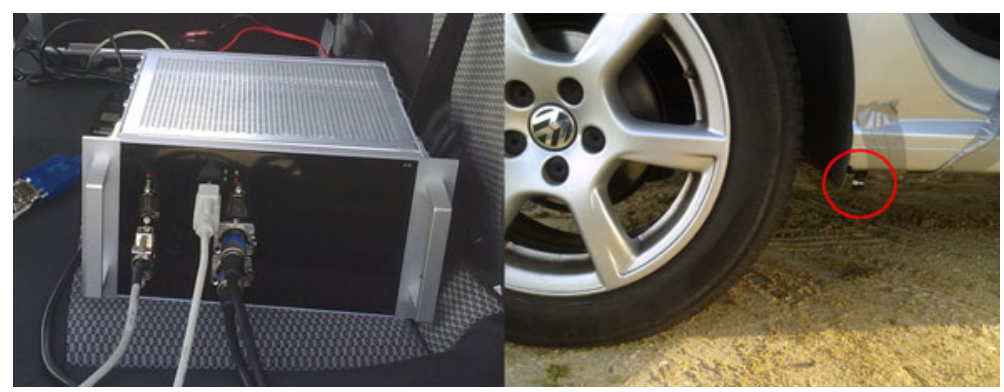


derecha de esta figura, se observa una de las posibles ubicaciones del micrófono de pruebas. Todos los algoritmos de procesado de señal necesarios para la captura de audio y extracción de características, así como el clasificador, han sido implementados sobre el DSP que incorpora el prototipo, y son ejecutados por el mismo en tiempo real, utilizando una ínfima cantidad de sus recursos. El funcionamiento del sistema es óptimo, obteniéndose tasas de acierto muy similares a las que se obtuvieron con el método de grabación y postprocesado en Matlab, y la sensación subjetiva de los conductores sometidos a pruebas es la de que la detección de asfalto mojado se produce de manera instantánea y fidedigna.

El interfaz de conexión con las centralitas del vehículo, permite obtener diversas variables dinámicas del mismo, como la velocidad y las revoluciones de giro del motor. Estas variables, si bien no han sido utilizadas inicialmente, podrían ser introducidas también en el clasificador, para mejorar aún más la tasa de aciertos del mismo, especialmente para velocidades inferiores a $30 \mathrm{~km} / \mathrm{h}$. Este prototipo también incorpora un interfaz que permite su conexión opcional a un PC, para obtener datos acerca de su funcionamiento interno, como por ejemplo la velocidad obtenida directamente de la centralita del vehículo, y la salida sin filtrar del detector, tal y como se muestran en la Figura 5.

Dado que el prototipo inicial utiliza un DSP mucho más potente de lo necesario, actualmente se está trabajando en producir una versión mucho más reducida del sistema, utilizando un microcontrolador de propósito general. Esta versión podría integrarse sin problemas en las centralitas del vehículo, y tiene un coste mucho más reducido que la inicial.

Figura 5 Velocidad del vehículo y salida sin filtrar del detector durante una de las pruebas del sistema. La zona de asfalto mojado es cruzada desde alrededor del segundo 13,6 al segundo 18

\section{Conclusiones}

Se ha ideado una metodología para la detección del estado climático del asfalto, en base al análisis del ruido de rodadura generado durante la marcha del vehículo. La citada metodología incluye el pre-procesado de la señal acústica captada, y su aplicación a un clasificador basado en máquinas de vectores soporte (SVM).

La metodología desarrollada ha sido puesta a prueba utilizando una primera implementación en el entorno Matlab, obteniéndose tasas de acierto muy elevadas, por encima del $91 \%$ para la detección de asfalto seco y asfalto mojado. Esto ha llevado a desarrollar un prototipo hardware para la detección de estado del asfalto en tiempo real. En este prototipo se han implementado todos los algoritmos necesarios para el funcionamiento del sistema, obteniéndose resultados muy similares a los que inicialmente se registraron usando Matlab. Adicionalmente, los conductores que han evaluado el sistema subjetivamente han indicado que la detección del estado del asfalto se realiza de manera rápida y fiable.

El óptimo funcionamiento mostrado por el primer prototipo hardware, ha llevado al desarrollo de un segundo prototipo, en el que se está trabajando actualmente. Los objetivos de este segundo prototipo son la minimización del tamaño y coste del sistema. Adicionalmente se realizarán pruebas en asfalto nevado y helado, así como con un mayor abanico de tipos de neumáticos y asfaltos, para mejorar la generalización del sistema y para añadir los estados de asfalto helado y asfalto nevado a los contemplados por el clasificador.

La disponibilidad en el vehículo de un sistema que avise al conductor en tiempo real de las condiciones de

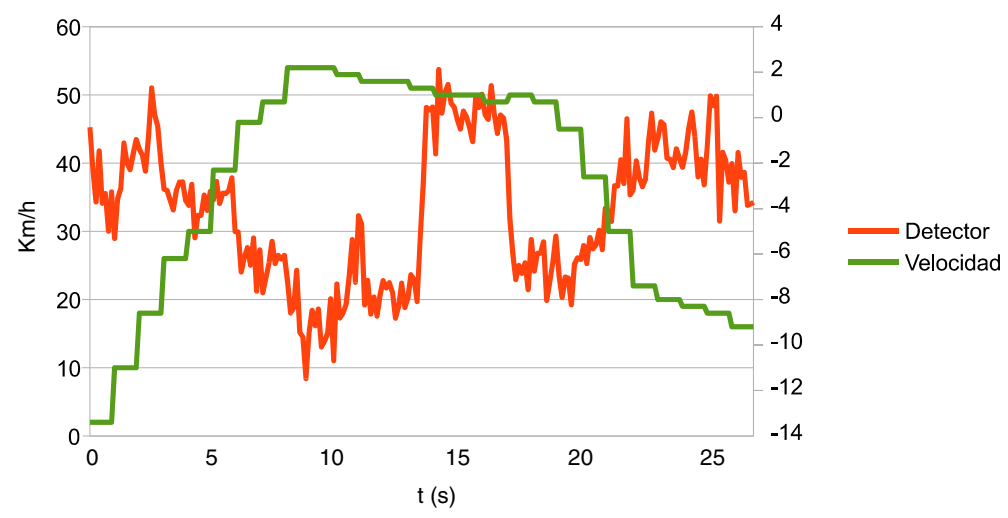


asfalto resbaladizo, y que el conductor perciba como fiable, se espera que tenga importantes implicaciones en la mejora de la seguridad, pudiendo evitar accidentes debidos a descuidos del conductor, o a fenómenos psicológicos que dificultan la percepción del estado del firme, como el efecto anclaje. Esta información también podría ser aprovechada por el subsistema de control de tracción, para que este se adapte al estado de la carretera, mejorando la seguridad y el confort.

\section{Referencias}

1. Einhorn, H. J., \& Hogarth, R. M. (1982). A theory of diagnostic inference. I. Imagination and the psychophysics of evidence [M]. Chicago Univ Il Center for Decision Research: Defense Technical Information Center.

2. Erke, A., Sagberg, F., \& Hagman, R. (2007). Effects of route guidance variable message signs (VMS) on driver behavior [J]. Transportation Research Part F: Traffic Psychology and Behaviour, 10(6), 447-457.
3. Guyon, I., Weston, J., Barnhill, S., \& Vapnik, V. (2002). Gene selection for cancer classification using support vector machines [J]. Machine Learning, 46(1), 389-422.

4. Norrman, J., Eriksson, M., \& Eriksson, M. (2000). Relationships between road slipperiness traffic accident risk and winter road maintenance activity [J]. Climate Research, 15(3), 185-193.

5. Rämä, P. (1999). Effects of weather-controlled variable speed limits and warning signs on driver behavior [J]. Transportation Research Record: Journal of the Transportation Research Board, 1689(-1), 53-59.

6. Rämä, P. (1999). Effects of weather-controlled variable speed limits and warning signs on driver behavior [J]. Transportation Research Record: Journal of the Transportation Research Board, 1689(1), 53-59.

7. Theodoridis, S., \& Koutroumbas, K. (2009). Pattern recognition [M]. Elsevier/Academic Press. ISBN 9781597492720.

8. Wallman, C. G. (1997). Driver behaviour on winter roads: A driving simulator study [M]. VTI Rapport 419A. Swedish National Road and Transport Research Institute.

9. Weston, J., Elisseeff, A., Schölkopf, B., \& Tipping, M. (2003). Use of the zero norm with linear models and kernel methods [J]. The Journal of Machine Learning Research, 3, 1439-1461.

10. Weston, J., Elisseeff, A., BakIr, G., \& Sinz, F. (2006). The spider. $<$ http://people.kyb.tuebingen.mpg.de/spider/\&gt. 